\title{
ERRATUM
}

\section{The lateral femoral cutaneous nerve canal}

TO THE READERSHIP: Errors appeared in the article by Hanna (Hanna A: The lateral femoral cutaneous nerve canal. J Neurosurg [epub ahead of print April 22, 2016; DOI: 10.3171/2016.1.JNS152262]).

Due to an administrative error, the legends for 3 figures are missing the credit line attributing copyright to the author. The updated legends and figures are shown here.
The article has been corrected online as of November 4, 2016.

Gillian Shasby
Director of Publications-Operations
Journal of Neurosurgery Publishing Group, Charlottesville, VA

CORRESPONDING ARTICLE See pp 972-978.

INCLUDE WHEN CITING

Published online November 4, 2016; DOI: 10.3171/2016.10.JNS152262a.

(c)AANS, 2017
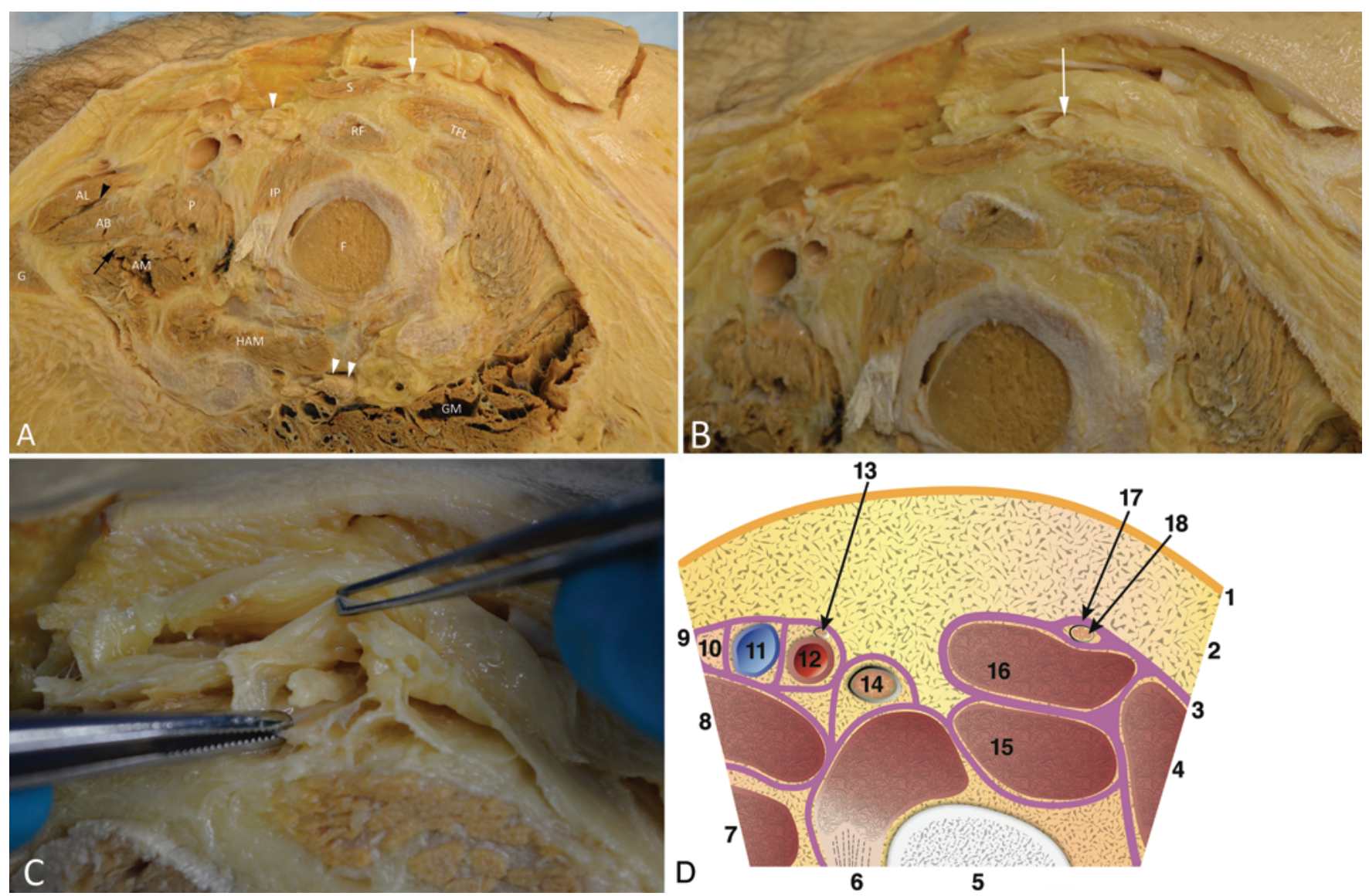

FIG. 6. Oblique axial cut of the left thigh caudal to the ASIS, showing the LFCN in its individual canal. A: White arrow = LFCN in its own canal; single white arrowhead = femoral nerve; double arrowheads = sciatic nerve; black arrow = obturator nerve (posterior division); black arrowhead = obturator nerve (anterior division); $\mathrm{AB}=$ adductor brevis; $\mathrm{AL}=$ adductor longus; $\mathrm{AM}=$ adductor magnus; $\mathrm{F}$ = femur; $\mathrm{G}$ = gracilis; $\mathrm{GM}=$ gluteus maximus; $\mathrm{HAM}=$ hamstrings; $I \mathrm{P}=$ iliopsoas; $\mathrm{P}$ = pectineus; $\mathrm{RF}=$ rectus femoris; $S=$ sartorius; TFL = tensor fasciae latae. B: Close-up view, with the white arrow pointing to the LFCN in its individual canal. C: Close-up view, with 2 forceps holding the anterior and posterior layers of the LFCN canal. D: Diagrammatic representation of the left LFCN canal in an axial cut. 1 = skin; 2 = subcutaneous tissue; 3 = fascia lata; 4 = tensor fasciae latae; 5 = femur; 6 = iliopsoas; 7 = adductor magnus; 8 = pectineus; 9 = femoral sheath; 10 = femoral canal; $11=$ femoral vein; $12=$ femoral artery; $13=$ femoral branch of genitofemoral nerve; 14 = femoral nerve; 15 = rectus femoris; $16=$ sartorius; $17=\mathrm{LFCN}$ canal; $18=\mathrm{LFCN}$. Copyright Amgad Hanna (Panel D). Published with permission. 

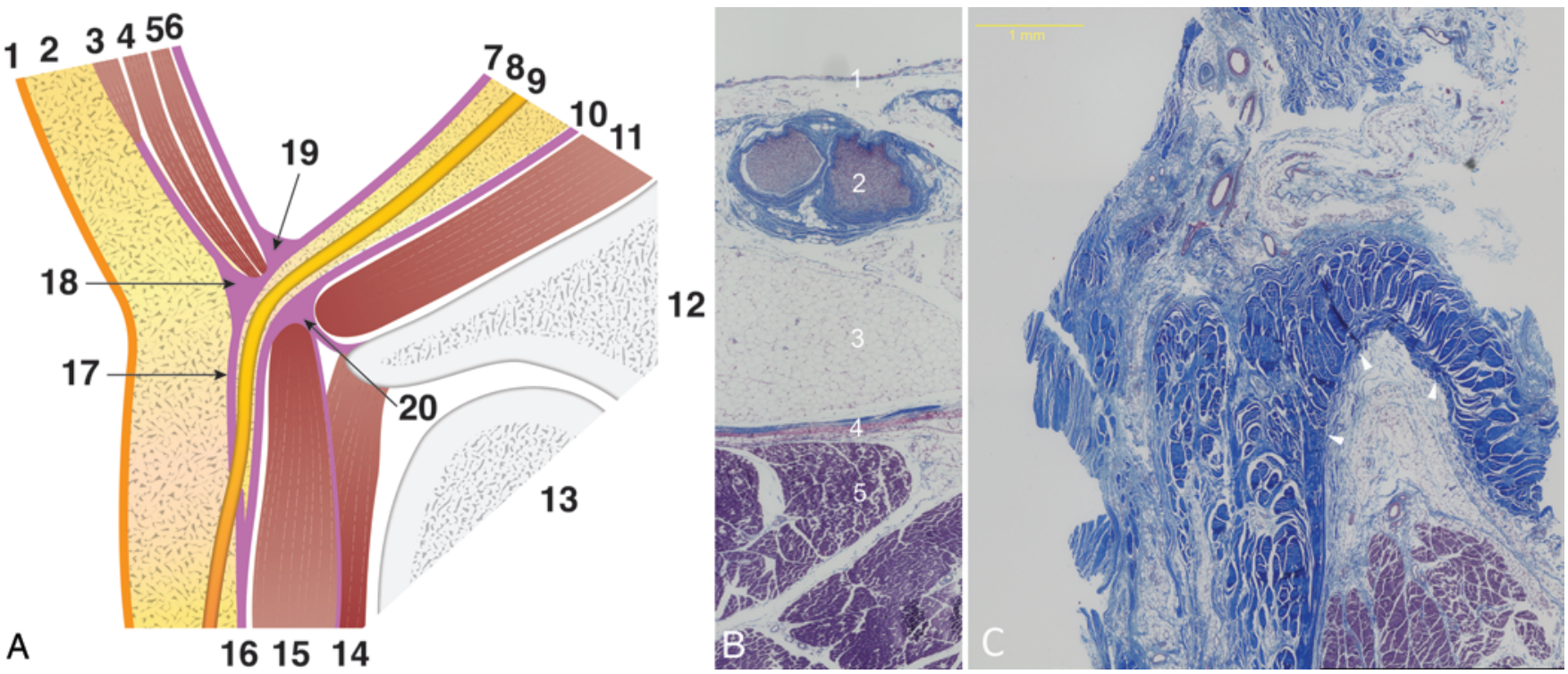

FIG. 8. A: Diagrammatic representation of the LFCN canal in an oblique sagittal view at the level of the anterior inferior iliac spine. 1 = skin; 2 = subcutaneous tissue; 3 = external oblique muscle and aponeurosis; 4 = internal oblique; $5=$ transversus abdominis; 6 = transversalis fascia; 7 = fascia iliaca superficial lamina; $8=$ retroperitoneal fat; $9=$ LFCN; 10 = fascia iliaca deep lamina; $11=$ iliacus; 12 = iliac bone; 13 = femur; 14 = rectus femoris; 15 = sartorius; 16 = fascia lata; $17=$ LFCN canal; $18=$ inguinal ligament; $19=$ iliopubic tract; 20 = thickening of the fascia deep to the LFCN (here by the tendinous origin of the sartorius; can also be a slip from the inguinal ligament, or periosteum if the nerve is riding over the ASIS or iliac crest). B: Axial section through the retroperitoneal segment of the LFCN. 1 = anterior lamina of fascia iliaca; 2 = LFCN; 3 = retroperitoneal fat; 4 = posterior lamina of fascia iliaca; $5=$ iliacus muscle. C: Axial section through the floor of the LFCN canal (arrowheads), showing a thick layer separating the nerve from the muscle in the region of the inguinal ligament. Trichrome stain (B and C), no magnification (B). Copyright Amgad Hanna (Panel A). Published with permission.

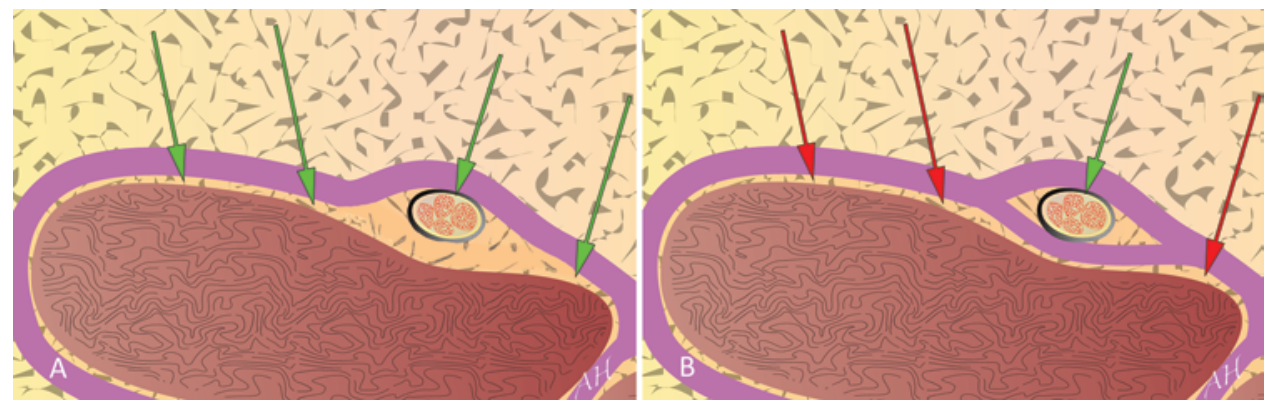

FIG. 10. A: Wrong concept illustrating a single layer of fascia superficial to the LFCN. Based on this, opening the fascia in any location will allow access to the nerve (green arrows). B: Correct concept illustrating the LFCN canal with its 2 components superficial and deep to the nerve. Based on this, opening the fascia on top of the nerve is the only way to find the nerve (green arrow). Opening on either side of the nerve canal (red arrows), not only will miss the nerve but also will make the search for the nerve extremely difficult because the second deeper layer is still intact and shields the nerve from the surgeon's eyes. Copyright Amgad Hanna. Published with permission. 